\title{
Association between Meibomian Gland Dysfunction and Depressive Symptoms: A Multi-center Cross- sectional Study
}

\section{Zhenyu Wei}

Beijing Institute of Ophthalmology, Beijing Tongren Eye Center, Capital Medical University Junqi Liang

University of Central Lancashire

\section{Kai Cao}

Beijing Institute of Ophthalmology, Beijing Tongren Eye Center, Capital Medical University

\section{Leying Wang}

Beijing Institute of Ophthalmology, Beijing Tongren Eye Center, Capital Medical University

\section{Christophe Baudouin}

Quinze-Vingts National Ophthalmology Hospital, IHU FOReSIGHT, Paris and Versailles Saint- Quentin-en-

Yvelines University

\section{Antoine Labbé}

Quinze-Vingts National Ophthalmology Hospital, IHU FOReSIGHT, Paris and Versailles Saint- Quentin-enYvelines University

Qingfeng Liang ( $\sim$ lqflucky@163.com )

Beijing Institute of Ophthalmology, Beijing Tongren Eye Center, Capital Medical University

\section{Research Article}

Keywords: Meibomian gland dysfunction, Depression, Risk-factors, Correlation

Posted Date: September 28th, 2021

DOI: https://doi.org/10.21203/rs.3.rs-923660/v1

License: (c) (i) This work is licensed under a Creative Commons Attribution 4.0 International License. Read Full License 


\section{Abstract}

Increased prevalence of depression has been found in patients with meibomian gland dysfunction (MGD); however, specific conditions of patients suffered from MGD and depression remains unclear. Our aim was to investigate the prevalence of depression in patients with MGD and analyze the risk factors of depression in MGD patients. In this multi-center cross-sectional study, we enrolled 830 MGD patients and 114 normal controls. Demographic information was collected by designed questionnaires about lifestyle habits, systemic and ocular medical history. Ophthalmic examinations were performed in a formal order. Depression symptoms were evaluated with a questionnaire survey using a modified self-rating depression scale (M-SDS). The correlations between the M-SDS score and demographic and clinical information were analyzed. The prevalence of hyperlipidemia and obstructive sleep apnea-hypopnea syndrome (OSAHS) was higher in the MGD group. There were 78 cases (9.4\%) with depressive symptoms in the MGD group whereas there were 4 cases (3.5\%) in controls. Similarly, M-SDS was higher in the MGD group. The characteristics of depression in the MGD group included: crying spells, sleep disturbance and depressed appetite. Some systemic diseases (OSAHS, allergy, skin disease) and the use of contact lenses was correlated with an increased risk of depressive symptoms in MGD patients.

\section{Introduction}

Meibomian gland dysfunction (MGD) is a chronic, diffuse abnormality of the meibomian gland, which can induce the alteration of tear film lipids, a decrease of tear film stability and symptoms of eye irritation. As a leading cause of dry eye disease (DED), MGD becomes a major public ocular health problem, which prevalence rate varies from $3.5 \%$ to almost $70 \% 1,2$. Its occurrence is always related to gender, age, and race, and a strikingly higher prevalence appeared in the Asia population (46.2-69.3\%) ${ }^{3-}$ 5 . In recent years, with the change of social lifestyle and the increase of video display terminal (VDT) viewing, the number of MGD patients has increased significantly ${ }^{6}$.

Clinically, persistent ocular surface discomfort from MGD, including ocular irritation, dryness, burning sensation, and blurring of vision, could always produce a negative impact on life quality. Notably, these symptoms from MGD may be overlap or very similar to those reported in DED patients ${ }^{7}$. Some studies reported the relationship between DED and psychiatric alteration. Especially, Inomata et al ${ }^{5}$ used Dry Eye Rhythm to collect real-world data and found that severe DE symptoms were correlated with an increased risk of depressive symptoms. This relationship was also confirmed by a meta-analysis ${ }^{8}$, which found a higher depression prevalence in DED patients (29\%) than that in controls.

Although MGD becomes a leading cause of DED, few studies attempted to explore the relationship between mental health and MGD. Especially, a potential concern is that chronic symptoms from MGD could induce a negative impact on mental health and the treatment effect of MGD cannot be completely satisfactory ${ }^{9}$. Therefore, it is important to undertake a large-sample study to investigate the MGD patients' mental alteration to make clear the relationship between MGD and depression. With this multi- 
center epidemic research, we hope to find the risk factors of depressive symptoms in MGD cases and achieve the purpose of early detection and intervention of depression in patients with MGD.

\section{Results}

\section{Patient characteristics}

A total of 944 participants were included: 830 patients with MGD and 114 control subjects. The mean age was $42.6 \pm 13.2$ years (range, $18-87$ years) in the MGD group and $40.3 \pm 14.6$ years (range, $18-76$ years) in the control group. The number of women in the MGD group and control group was $540(65.0 \%)$ and 84 (73.7\%), respectively. There was no significant difference in age $(P=0.057)$, gender $(P=0.068)$ and living area (urban or rural, $P=0.188$ ) between these two groups.

The demographic data and basic medical history of MGD patients and normal controls are summarized in Table 1. The survey of systemic diseases showed that the MGD group had higher prevalence of hyperlipidemia ( $8.8 \%$ vs. $0.8 \%, P=0.003$ ) and OSAHS (obstructive sleep apnea-hypopnea syndrome) ( $15.9 \%$ vs. $7.9 \%, P=0.024)$ compared with controls. There was no significant difference for other disease prevalence (hypertension, diabetes mellitus, coronary heart disease, cerebral infarction, allergy, skin disease) within the two groups. Similarly, there was no difference in ocular medical history between the two groups (diabetic retinopathy, glaucoma, cataract, ametropia and contact lens wear). 
Table 1

Characteristics of MGD patients and normal controls

\begin{tabular}{|c|c|c|c|}
\hline Parameters & $\begin{array}{l}\text { Normal controls } \\
(n=114)\end{array}$ & $\begin{array}{l}\text { MGD group } \\
(n=830)\end{array}$ & $P$ value \\
\hline Age & $40.3 \pm 14.6$ & $42.6 \pm 13.2$ & 0.057 \\
\hline \multicolumn{4}{|l|}{ Gender [n (\%)] } \\
\hline Male & $30(26.3 \%)$ & $290(34.9 \%)$ & \multirow[t]{2}{*}{0.068} \\
\hline Female & $84(73.7 \%)$ & $540(65.1 \%)$ & \\
\hline \multicolumn{4}{|l|}{ Residence [n (\%)] } \\
\hline Urban & $107(94.0 \%)$ & 747 (90.0\%) & \multirow[t]{2}{*}{0.188} \\
\hline Rural & $7(6.0 \%)$ & $83(10.0 \%)$ & \\
\hline \multicolumn{4}{|l|}{ Systemic diseases [n (\%)] } \\
\hline Hypertension & $10(8.8 \%)$ & $73(8.8 \%)$ & 0.993 \\
\hline Hyperlipidemia & $1(0.8 \%)$ & $73(8.8 \%)$ & $0.003^{*}$ \\
\hline Diabetes mellitus & $3(2.6 \%)$ & $25(3.0 \%)$ & 1.000 \\
\hline Coronary heart disease & $0(0.0 \%)$ & $9(1.1 \%)$ & 0.610 \\
\hline Cerebral infarction & $0(0.0 \%)$ & $5(0.6 \%)$ & 1.000 \\
\hline OSAHS & $9(7.9 \%)$ & $132(15.9 \%)$ & $0.024^{\star}$ \\
\hline Allergy & $10(8.8 \%)$ & $79(9.5 \%)$ & 0.798 \\
\hline Skin disease & $5(4.82 \%)$ & $43(5.18 \%)$ & 0.717 \\
\hline \multicolumn{4}{|l|}{ Ocular diseases [n (\%)] } \\
\hline Diabetic retinopathy & $1(0.8 \%)$ & $3(0.3 \%)$ & 0.403 \\
\hline Glaucoma & $0(0.0 \%)$ & $1(0.1 \%)$ & 1.000 \\
\hline Cataract & $8(7.0 \%)$ & $48(5.8 \%)$ & 0.601 \\
\hline Ametropia & $92(63.9 \%)$ & $500(60.2 \%)$ & 0.408 \\
\hline Contact lens wear & $4(3.5 \%)$ & $27(3.25 \%)$ & 0.782 \\
\hline
\end{tabular}

In the MGD group, 86 (10.4\%) cases smoked and their smoking index was $159.3 \pm 26.8$, which were higher than that in normal controls $(5.3 \%, 73.2 \pm 12.1$; all $P \otimes 0.05)$. In addition, one-fifth of MGD cases always 
drank alcohol (drinking index $325.2 \pm 45.3$ ), higher than the control group (all $P \otimes 0.05$ ). Although the duration of sport and walking every day in these two groups were no significant different, the reading time in MGD (60\% cases, almost every day), longer than that in the control group (48.2\%, almost every day, $P=0.029$, Table 2). 
Table 2

Lifestyle habits of MGD patients and normal controls

\begin{tabular}{|c|c|c|c|}
\hline Parameters & $\begin{array}{l}\text { Normal controls } \\
(n=114)\end{array}$ & $\begin{array}{l}\text { MGD group } \\
(n=830)\end{array}$ & $P$-value \\
\hline \multicolumn{4}{|l|}{ Smoking } \\
\hline Number of Smokers [n (\%)] & $6(5.3 \%)$ & $86(10.4 \%)$ & \multirow[t]{2}{*}{$0.041^{*}$} \\
\hline Number of Non- Smokers [n (\%)] & $108(94.7 \%)$ & $744(89.6 \%)$ & \\
\hline Smoking index of smokers ${ }^{\#}$ & $73.2 \pm 12.1$ & $159.3 \pm 26.8$ & $0.032^{*}$ \\
\hline Drinking & & & 0.745 \\
\hline Number of Drinkers & $9(7.9 \%)$ & $172(20.8 \%)$ & \multirow[t]{2}{*}{$0.001^{\star}$} \\
\hline Number of Non-Drinkers & $105(92.1 \%)$ & $658(79.2 \%)$ & \\
\hline Alcohol drinking index of drinkers ${ }^{\#}$ & $283.5 \pm 22.3$ & $325.2 \pm 45.3$ & 0.496 \\
\hline \multicolumn{4}{|l|}{ Reading [n (\%)] } \\
\hline 0 (never) & $0(0.0 \%)$ & $32(3.9 \%)$ & \multirow[t]{5}{*}{0.029} \\
\hline 1 (almost never) & $22(19.3 \%)$ & $100(12.1 \%)$ & \\
\hline 2 (1-2day/week) & $14(12.3 \%)$ & $87(10.5 \%)$ & \\
\hline 3 (3-5day/week) & $23(20.2 \%)$ & $113(13.6 \%)$ & \\
\hline 4 (almost every day) & $55(48.2 \%)$ & $498(60.0 \%)$ & \\
\hline \multicolumn{4}{|l|}{ Sports (hours / week) } \\
\hline Low intensity & $3.6 \pm 2.2$ & $2.6 \pm 1.4$ & 0.092 \\
\hline Medium intensity & $2.0 \pm 1.2$ & $2.3 \pm 2.0$ & 0.404 \\
\hline High intensity & $1.3 \pm 1.1$ & $1.0 \pm 1.2$ & 0.903 \\
\hline \multicolumn{4}{|l|}{ Walks (> 30mins) [n (\%)] } \\
\hline 0 (never) & $74(65.0 \%)$ & $521(62.7 \%)$ & \multirow[t]{4}{*}{0.280} \\
\hline 1 (2-3days/week) & $11(9.6 \%)$ & $125(15.6 \%)$ & \\
\hline 2 (4-5days/week) & $26(22.8 \%)$ & $144(17.3 \%)$ & \\
\hline 3 (6-7days/week) & $3(2.6 \%)$ & $40(4.8 \%)$ & \\
\hline
\end{tabular}

Note: ${ }^{*} P<0.05$ was considered statistically significant. \# Smoking and drinking index were calculated as follows: Smoking index = (number of cigarettes consumed per day) $\times$ (years of smoking); Alcohol drinking index $=($ Gram of Liquor consumed per day) $\times$ (years of drinking). 


\begin{tabular}{|llll|}
\hline Parameters & $\begin{array}{l}\text { Normal controls } \\
(\mathbf{n = 1 1 4 )}\end{array}$ & $\begin{array}{l}\text { MGD group } \\
(\mathbf{n = 8 3 0 )}\end{array}$ & P-value \\
\hline Inactive time (hours / day) & $5.6(3.0-7.5)$ & $4.7(3.6-6.2)$ & 0.442 \\
\hline $\begin{array}{l}\text { Note: } * P<0.05 \text { was considered statistically significant. \# Smoking and drinking index were calculated } \\
\text { as follows: Smoking index= (number of cigarettes consumed per day) } \times(\text { years of smoking); Alcohol } \\
\text { drinking index= (Gram of Liquor consumed per day) } \times \text { (years of drinking). }\end{array}$ & \\
\hline
\end{tabular}

\section{Clinical manifestations}

Ocular examination revealed no significant difference between MGD patients and normal controls in BCVA and IOP $(P=0.451,0.078)$. Compared with normal controls, MGD patients had higher OSDI score ( $26.0 \pm 15.1$ vs. $6.5 \pm 4.8, P<0.001)$, shorter TBUT ( $4.1 \pm 2.3$ s vs. $7.3 \pm 4.2 \mathrm{~s}, P=0.002)$, lower LLT ( $51.7 \pm$ $24.9 \mathrm{~nm}$ vs. $67.9 \pm 21.9 \mathrm{~nm}, P=0.004)$ and a higher Oxford scale $(0.7 \pm 1.2$ vs. $0.2 \pm 0.6, P=0.012)$. In the MGD group, the score of the lid margin and the loss rate of meibomian glands were higher than normal controls ( $2.9 \pm 0.8$ vs. $1.2 \pm 0.7,34.5 \pm 12.2$ vs. $11.5 \pm 11.9$; all $P=0.002)$. Meanwhile, the higher score of MG expressibility ( $1.7 \pm 0.1$ vs. $0.6 \pm 0.1, P=0.001)$ and Meibum ( $2.2 \pm 0.2$ vs. $0.5 \pm 0.2, P=0.010)$ were also detected in the MGD group, comparing with normal controls (Table 3 ). 
Table 3

Ocular surface evaluation in normal controls and MGD group

\begin{tabular}{|c|c|c|c|}
\hline Parameters & $\begin{array}{l}\text { Normal controls } \\
(n=114)\end{array}$ & $\begin{array}{l}\text { MGD group } \\
(n=830)\end{array}$ & $P$-value \\
\hline BCVA & $0.9 \pm 0.2$ & $0.9 \pm 0.2$ & 0.451 \\
\hline IOP $(\mathrm{mmHg})$ & $14.0 \pm 2.9$ & $13.2 \pm 2.5$ & 0.078 \\
\hline OSDI & $6.5 \pm 4.8$ & $26.0 \pm 15.1$ & $<0.001^{*}$ \\
\hline TBUT (s) & $7.3 \pm 4.2$ & $4.1 \pm 2.3$ & $0.002^{*}$ \\
\hline Schirmer I test (mm) & $11.5 \pm 9.4$ & $10.1 \pm 8.0$ & 0.250 \\
\hline Lipid layer thickness (nm) & $67.9 \pm 21.9$ & $51.7 \pm 24.9$ & $0.004^{\star}$ \\
\hline Cornea staining & $0.2 \pm 0.6$ & $0.7 \pm 1.2$ & $0.012^{\star}$ \\
\hline MG loss (\%) & $11.5 \pm 11.9$ & $34.5 \pm 12.2$ & $0.002^{\star}$ \\
\hline Rate of partial blinking & $0.6 \pm 0.3$ & $0.6 \pm 0.4$ & 0.634 \\
\hline Lid margin abnormality score & $1.2 \pm 0.7$ & $2.9 \pm 0.8$ & $0.002^{\star}$ \\
\hline MG expressibility score & $0.6 \pm 0.1$ & $1.7 \pm 0.1$ & $0.001^{\star}$ \\
\hline Meibum score & $0.5 \pm 0.2$ & $2.2 \pm 0.2$ & $0.010^{\star}$ \\
\hline
\end{tabular}

\section{Depression analysis}

In this study, M-SDS was used to evaluate depression and its severity. The score of M-SDS in the MGD group was $30.7 \pm 7.7$, significantly higher than that in normal controls $(27.9 \pm 6.2, P=0.001)$. There were 78 cases $(9.4 \%)$ with depressive symptoms in the MGD group, which was higher than that in controls (4 cases, $3.5 \%)(P=0.036)$. Among MGD cases with depression symptoms, most of them appeared mild (49/78) and moderate (24/78) depressive symptoms. Between normal controls and the MGD group, the severity of depression symptoms was no significant difference $(P=0.517$, Table 4$)$. 
Table 4

Evaluation of depression symptom in normal controls and MGD group

\begin{tabular}{|llll|}
\hline Parameters & Normal controls $(\mathbf{n = 1 1 4 )}$ & MGD group $(\mathbf{n = 8 3 0 )}$ & $\boldsymbol{P}$ value \\
\hline Modified self-rating depression scale & $27.9 \pm 6.2$ & $30.7 \pm 7.7$ & $0.001^{*}$ \\
\hline Without depression [n (\%)] & $110(96.5 \%)$ & $752(90.6 \%)$ & $0.036^{*}$ \\
\hline With depression [n (\%)] & $4(3.5 \%)$ & $78(9.4 \%)$ & \\
\hline Mild Depression & $4(3.5 \%)$ & $49(5.9 \%)$ & \\
\hline Moderate Depression & $0(0)$ & $24(2.9 \%)$ & \\
\hline Severe Depression & $0(0)$ & $5(0.6 \%)$ & \\
\hline $\begin{array}{l}\text { Note: Depression was assessed using the Self-rating scale and definite depression was defined as } \\
\text { having a depression score of 40 or higher. The severity of depression was rated as mild, 40-50 } \\
\text { scores; moderate, 50-60 scores; and severe, above 60 scores. *P<0.05 was considered statistically } \\
\text { significant. }\end{array}$ & & \\
\hline
\end{tabular}

After analyzing each result of the 17 questions in the M-SDS questionnaire, three parameters (crying spells, sleep disturbance and depressed appetite) had a higher score in the MGD group with depression symptoms, comparing with normal controls with depression (P凶0.001, 0.012, 0.008, Table 5). 
Table 5

Analysis of M-SDS questionnaire survey among depression cases

\begin{tabular}{|llll|}
\hline Questions & $\begin{array}{l}\text { Normal controls } \\
(\mathbf{n}=\mathbf{7 8})\end{array}$ & $\begin{array}{l}\text { MGD group } \\
(\mathbf{n}=\mathbf{4})\end{array}$ & Pvalue \\
\hline Depressed affect & $1.75 \pm 0.96$ & $2.21 \pm 0.89$ & 0.322 \\
\hline Diurnal variation & $2.25 \pm 0.50$ & $2.44 \pm 1.09$ & 0.736 \\
\hline Crying spells & $1.00 \pm 0.00$ & $1.46 \pm 0.73$ & $<0.001^{*}$ \\
\hline Sleep disturbance & $1.25 \pm 0.50$ & $2.68 \pm 1.10$ & $0.012^{\star}$ \\
\hline Depressed appetite & $1.25 \pm 0.50$ & $2.87 \pm 1.19$ & $0.008^{\star}$ \\
\hline Fear of blindness & $2.75 \pm 1.26$ & $3.19 \pm 1.08$ & 0.431 \\
\hline Weight loss & $2.00 \pm 0.82$ & $1.58 \pm 0.85$ & 0.331 \\
\hline Constipation & $1.25 \pm 0.50$ & $1.85 \pm 1.03$ & 0.257 \\
\hline Tachycardia & $1.50 \pm 0.58$ & $1.90 \pm 0.77$ & 0.311 \\
\hline Fatigue & $2.25 \pm 1.26$ & $2.56 \pm 1.04$ & 0.561 \\
\hline Confusion & $3.50 \pm 1.00$ & $2.74 \pm 1.00$ & 0.144 \\
\hline Psychomotor retardation & $2.50 \pm 1.29$ & $2.49 \pm 1.14$ & 0.983 \\
\hline Irritability & $3.00 \pm 1.15$ & $2.47 \pm 0.88$ & 0.253 \\
\hline Hopelessness & $1.75 \pm 0.50$ & $2.60 \pm 1.00$ & 0.095 \\
\hline Agitation & $2.25 \pm 1.50$ & $2.54 \pm 0.89$ & 0.544 \\
\hline Indecisiveness & $2.00 \pm 1.41$ & $2.26 \pm 1.04$ & 0.636 \\
\hline Personal devaluation & $3.00 \pm 1.41$ & $2.78 \pm 1.03$ & 0.685 \\
\hline Suicidal ideas & $3.00 \pm 1.15$ & $2.46 \pm 0.95$ & 0.276 \\
\hline Dissatisfaction & $2.50 \pm 1.73$ & $1.67 \pm 1.05$ & 0.138 \\
\hline Emptiness & $2.50 \pm 1.29$ & $2.72 \pm 0.99$ & 0.673 \\
\hline Note: ${ }^{*}>0.05$ was considered statistically significant. & \\
\hline & & & \\
\hline
\end{tabular}

In the MGD group, relevant risk factors for depressive symptoms were identified by univariate analysis of odds ratios and were further evaluated by multivariate analysis. The logistic regression analysis showed that the significant univariate odds ratios were $1.95(1.02,3.71)$ for living place, $6.57(1.08,39.94)$ for the history of cerebral infarction, $1.79(1.08,2.97)$ for the history of OSAHS, $3.40(1.89,6.12)$ for the history of allergy, $4.82(2.39,9.68)$ for the history of skin disease, $3.61(1.48,8.83)$ for contact lens wearing, 1.33 $(1.07,1.65)$ for inactive time per day, $4.76(1.19,18.99)$ for BCVA, and $1.01(1.00,1.03)$ for OSDI. The 
significant multivariate odds ratios of covariates were as follows: $1.79(1.00,3.18)$ for OSAHS, $2.08(1.03$, 4.22) for allergy, $3.41(1.48,7.90)$ for skin disease, $1.14(1.04,1.25)$ for inactive time, and $4.52(1.60$, 12.75) for contact lens wearing (all $P<0.05$, Supplementary Table 1 ).

\section{Discussion}

MGD had a high prevalence worldwide, and it is higher in Asians than in Caucasians ${ }^{10-12}$. As a leading cause of DED, MGD often presents chronic ocular health problems, especially eye pain, foreign body, and alterations of optical quality, etc. It not only can adversely affect anyone's life quality but also the mental health of patients. Moreover, depression symptoms also strengthen the subjective feeling about ocular surface discomfort and always coexist with other health conditions. Till now the risk factors and the correlation between MGD and depression remains unclear. Based on our study, the prevalence of depression in the MGD group was higher than normal controls. Risk factors such as OSAHS, allergy, skin disease, contact lens wearing, and inactive time were correlated with the depression symptoms of MGD patients. The results enabled early detection of the signs of mental changes in the MGD group and offer the appropriate support promptly.

In this study, the prevalence of depression in the MGD group was $9.3 \%$, which is significantly higher than that in normal controls (3.5\%). As a major cause of DED, MGD can increase the evaporation of tear and decrease the tear volume, which induce short tear breakup times and tear hyperosmolarity. Some studies declared that hyperosmolarity could cause ocular surface inflammation and/or nerve damage, which may increase symptoms by peripheral sensitization. With functional magnetic resonance imaging (fMRI), Yu et al ${ }^{13}$ found regional homogeneity (ReHo) values of the middle frontal gyrus, inferior frontal gyrus, and superior frontal gyrus were significantly lower in dry eye patients compared to healthy controls. Symptoms of ocular surface injury in DED, especially MGD patients are associated with dysfunction in specific brain regions. And rianopoulou et $\mathrm{al}^{14}$ investigated brain function and microstructural changes in primary Sjögren syndrome (pSS) and found the functional connectivity abnormalities of the somatosensory cortex and microstructural abnormalities appeared in pSS, which were more pronounced in depression. In addition, depression could also increase the symptom of MGD. Mahmut et $\mathrm{al}^{15}$ invited 40 newly diagnosed depression patients and found that patients without a history of psychiatric drug use showed dry eye symptoms. Some studies revealed that negative emotional states would induce or enhance the perception of pain and irritation ${ }^{16}$. And the symptoms of pain, dryness, itchiness, stinging, foreign body sensation and sensitivity to light and wind from MGD can also negatively impact the mood of patients and have potential consequences of depression. So, patients with dry eye symptoms but no signs would have the lowest happiness scores. Subjective happiness scores in DED cases were found to be inversely correlated with dry eye symptoms ${ }^{17}$. At last, several studies have confirmed antidepressants may play an important role in provoking DED. Emel et $\mathrm{al}^{18}$ found that selective serotonin reuptake inhibitors (SSRIs) and serotonin-norepinephrine reuptake inhibitors increased the risk for eye dryness. Furthermore, Zhang et al ${ }^{19}$ proved that SSRIs could aggravate DED by activating the NF-KB pathway, which shows the interaction between dry eye and depression. Therefore, the depressive symptom could 
be alleviated by applying the managing MGD, which open the options of cooperative therapy between Ophthalmologists and psychiatrists to MGD cases.

Many risk factors make MGD patients more prone to develop depression, for instance, living in rural areas could be associated with depression in our study. However, results from a Korean elder cross-sectional study showed that living in the urban area is also strongly correlated with depression $(P<0.001)^{3}$. The economical differences between developed countries and developing countries may provide a reasonable explanation. Wang et al ${ }^{20}$ conducted a study about socio-demographic characteristics of depressive symptoms and found that educational attainment, the economic level had a significant association between depressive symptoms, which may explain the different results between Chinese and Korean studies.

Moreover, our study found that some systemic diseases (OSAHS, allergy, skin disease) were also related to depression. The results of the current study are consistent with previous results ${ }^{21,22}$. A meta-analysis by Jennifer et al concluded that the risk of developing depressive disorders was two to three times more likely among individuals with two or more chronic conditions ${ }^{23}$. Just as MGD, OSAHS, allergy and skin disease are always chronic and incurable diseases. Chronic pain and irritation are associated with sleep deficiency, activity and mobility limitations, social withdrawal, and loneliness. All these negative effects will induce cognitive impairment, loss of self-confidence and self-esteem, even anxiety and depression ${ }^{24}$.

Wearing a contact lens could also be argued to have potential adverse towards the development of depression. Patients with MGD who also wears contact lenses revealed an increase in dry eye symptom and ocular surface staining ${ }^{25,26}$. Consequently, the shorter TBUT result suggests that MGD and contact lens both contributes to the negative outcome of tear film integrity. Therefore, contact lens maybe induces depressive symptoms in MGD cases due to the aggravation of dry eye symptoms.

We have applied the M-SDS questionnaire to compared and analyzed some characteristics of depression between the MGD group and controls. For depressive patients in the MGD group, they presented with frequent crying, sleep disturbance and depressed appetite. It is still unclear about the reason causing the repeated crying but trying to get more tear secretion maybe a way to relieve mental tension in patients with MGD. Regarding decreased appetite previous study reported five patients with severe anorexia nervosa, all 5 patients complained of dry and irritated eye symptoms ${ }^{27}$, which indicate a possible causeeffect relationship between dry eye and appetite. Sherwin et al ${ }^{28}$ revealed that deficiency of vitamin $A$ is a significant risk factor of DED. Change of appetite can potentially decrease the level of intake of vitamin $A$ which then adversely affect depressive symptom. However, no clear evidence articulates the MGD or DED patients' eating habit changes.

Additionally, dry eye and insomnia influence each other in many ways. Masahiko et $\mathrm{al}^{29}$ reviewed 7 articles and concluded that dry eye patients suffer more from bad quality sleep. Wu et al ${ }^{30}$ also found that poor sleep quality may aggravate DED by affecting tear secretion and tear film stability, even indirectly aggravate depression at the same time. The result from the M-SDS questionnaire showed the 
difference in frequent crying, sleep disturbance and depressed appetite between MGD patients and control. Another interesting point is that depressive patients in the MGD group may more easily lose hope for their aspirations $(2.60 \pm 1.00$ vs. $1.75 \pm 0.50, P=0.095)$. In Van's study of patients with Sjögren's syndrome, these patients with a lower "cryability" had a higher score on frustration ${ }^{31}$. Gomes reported that the effect of the quality of life by dry eye may be underestimated ${ }^{32}$.

Our study offered insight into the importance of early detection and intervention of depressive disorder among MGD patients. Questionnaire results regarding sleep, eat changing and cry intention could help ophthalmologists screening depression patients. Such early conversation with the patient can not only prevent further prognosis but also facilitate a speedy recovery.

Even though this study is a focused multi-center cross-sectional study, the sample size could have improved to have more generalizability. Second, alongside the M-SDS questionnaire, other means of measure for the participants mental status could be employed. Third, this study is a cross-sectional study, more robust follow-up evidence is favorable for a deeper understanding of the relationship between MGD and depression.

In conclusion, this study served a pioneering role in connecting MGD and depression. Ophthalmologists should be aware of the association between MGD and depression, better understand patients' mental changes and better treat MGD patients with depression. Furthermore, the correlation between MGD patients with depression and the changing of their cytokines, chemokine, and inflammatory factors could be fascinating to investigate further.

\section{Methods}

\section{Subjects}

A multi-center cross-sectional study was carried out in 23 eye centers with 830 MGD patients and 114 normal controls from outpatient clinics between October 2016 and October 2019 in China. This study was registered in the Chinese Clinical Trial Registry (Registration number: ChiCTR-RDD-17013855) and got ethical approval from the Medical Ethics Committee of Beijing Tongren Hospital (TREC-2016-KY021). All participants were informed of the aims of the study and provided written informed consent was obtained from all subjects according to the declaration of Helsinki.

According to the diagnosis criteria defined by the International Workshop ${ }^{33}$ on MGD in 2011, the inclusion criteria of MGD were as follows: (1) aged $\geq 18$ years; (2) presence of at least one subjective symptom, such as ocular fatigue, dryness, foreign body sensation, pain, burning sensation, itching, redness and visual fluctuation; (3) more than one lid margin abnormalities under slit-lamp examination: palpebral margin hyperemia, irregular lid margin, vascular engorgement, plugged meibomian gland orifices, and anterior or posterior replacement of the mucocutaneous junction. Participants with one or more of the following criteria were excluded: (1) eyelid and conjunctival scar; (2) ocular surface abnormalities that 
may affect the corneal integrity; (3) unwilling or unable to stop medicine that can cause or aggravate dry eye disease; (4) pregnant and lactating women.

The inclusion criteria of the control group were as follows: (1) age $\geq 18$ years; (2) no ocular discomfort; (3) no abnormalities of lid margin under slit lamp microscopy. Exclusion criteria included: (1) skin or conjunctival inflammation; (2) eyelid trauma or abnormalities; (3) damaged corneal integrity of any eye; (4) systemic diseases or medications that cause DED; (5) pregnant or lactating women.

\section{Ocular surface Evaluation}

Demographic and medical information were collected through a series of questionnaires based on the

Beijing Eye Study ${ }^{34,35}$. These questionnaires included lifestyle queries (reading, sport, walk, etc.), systemic and ocular medical history (hypertension, hyperlipidemia, diabetes mellitus, diabetic retinopathy, glaucoma, cataract, etc.). The smoking index and alcohol drinking index was evaluated by the composite indicators of daily intake (per day) and intake duration (years) ${ }^{36}$. Each subject also underwent a quantification of ocular surface symptoms with the Ocular Surface Disease Index (OSDI) questionnaire (range, 0-100). Then, the ophthalmic examinations were performed with the following order: bestcorrected visual acuity (BCVA), intraocular pressure (IOP), slit-lamp examination, lipid layer thickness (LLT) measurement, meibomian gland loss evaluation (MGL), partial blink (PB) evaluation, tear film break-up time (TBUT) measurement, corneal staining, and Schirmer I test (Fig. 1).

BCVA was assessed after automatic refractometry (Auto Refractometer AR-610, Nidek Co., Ltd, Tokyo, Japan). If uncorrected visual acuity was lower than 1.0 , subjective refractometry was additionally performed. IOP was measured by pneumotonometry by an ophthalmologist. A slit-lamp examination was carried out by an experienced ophthalmologist and lid abnormalities, meibomian gland dysfunction, corneal disorders were evaluated. The LLT, MGL were measured with the Lipiview IIß device (Tear science, Morrisville, NC, USA). With the auto-analysis and calculation of the detection process from the Lipiview $\| \circledast$ device, PB was also recorded. The loss ratio of the meibomian gland could be detected after inverted upper and lower lid with infrared imaging technology and ImageJ (National Institutes of Health) software ${ }^{37}$.

TBUT was measured using sterile fluorescein strips impregnated with $0.6 \mathrm{mg}$ fluorescein sodium (Alcon Laboratories, St. Louis, MO, USA). After applying $50 \mu \mathrm{L}$ of normal saline solution to the paper strip, it was touched to the inferior fornix. The interval between a complete blink and the appearance of the first dry spot was noted. TBUT was measured 3 times and the average was calculated. Corneal staining was recorded by Oxford scale degree under cobalt blue light of silt-lamp ${ }^{38}$. The Schirmer I test was performed without anesthesia, after having the patient's eyes closed for 5 minutes.

\section{Depression symptom Evaluation}

Depression symptoms were evaluated with a questionnaire survey using a modified self-rating depression scale (M-SDS), which was adapted from the Zung self-rated depression scale and had been applied in The Beijing Eye Study. ${ }^{35}$ Total scores (range 20-80) were counted by summing the results of 
each 9 positive questions and 11 negative questions. The 1 to 4 responses to the negative question and positive question using an inverted recording method from 4 to 1 . Definite depression was defined as having an M-SDS score of 40 or higher. The severity of depression was rated as mild (40 50 scores), moderate (50 60 scores), and severe (above 60 scores).

\section{Data analysis}

Statistical analysis was performed with R software (www.r-project.org). For each patient, the right eye was chosen for statistical analysis. Kolmogorov-Smirnov test was used for testing the normality of each variable. Mean values and standardized deviations were used to make the basic statistical description for normally distributed continuous variables, otherwise, median values and interquartile range (IQR) were used. Frequency and percentile were used to make a basic statistical description for categorical variables. An independent two-sample t-test was used to make the comparison of normally distributed continuous variables between the MGD group and control group, while the Wilcoxon rank-sum test was used to make the comparison for non-normally distributed continuous variables. A Chi-square test was used to make the comparison of categorical variables between the MGD group and the control group. Logistic regression analysis was used to explore the association between the M-SDS score, clinical indicators, and depression. The significance level was set to be 0.05 .

\section{Declarations}

\section{Acknowledgments}

We thank the patients for their permission to publish their information.

\section{Author Contributions}

ZW was the major contributor in writing the manuscript. ZW, LW and QL collected the clinical data. JL, CB, $\mathrm{AL}$ made a substantial contribution to the revision of the manuscript. $\mathrm{KC}$, analyzed and interpreted the data. QL, the corresponding author, was primarily responsible for the experimental design and revision of the paper.

\section{Competing Interests}

No conflicting relationship exists for any author.

\section{Data availability statement}

All data relevant to the study are included in the article or uploaded as supplemental information. 


\section{References}

1. Hassanzadeh, S., Varmaghani, M., Zarei-Ghanavati, S. \& Heravian Shandiz, J. \& Azimi Khorasani, A. Global Prevalence of Meibomian Gland Dysfunction: A Systematic Review and Meta-Analysis. Ocular immunology and inflammation, 29, 66-75 https://doi.org/10.1080/09273948.2020.1755441 (2021).

2. Alghamdi, Y. A. et al. Epidemiology of Meibomian Gland Dysfunction in an Elderly Population., 35, 731-735 https://doi.org/10.1097/IC0.0000000000000815 (2016).

3. Kim, K. W. et al. Association between depression and dry eye disease in an elderly population. Investigative ophthalmology \& visual science, 52, 7954-7958 https://doi.org/10.1167/iovs.11-8050 (2011).

4. van der Vaart, R., Weaver, M. A., Lefebvre, C. \& Davis, R. M. The association between dry eye disease and depression and anxiety in a large population-based study. American journal of ophthalmology, 159, 470-474 https://doi.org/10.1016/j.ajo.2014.11.028 (2015).

5. Inomata, T. et al. Association between dry eye and depressive symptoms: Large-scale crowdsourced research using the DryEyeRhythm iPhone application. The ocular surface, 18, 312-319 https://doi.org/10.1016/j.jtos.2020.02.007 (2020).

6. O'Neil, E. C., Henderson, M., Massaro-Giordano, M. \& Bunya, V. Y. Advances in dry eye disease treatment. Curr Opin Ophthalmol, 30, 166-178 https://doi.org/10.1097/ICU.0000000000000569 (2019).

7. Nichols, K. K. et al. The international workshop on meibomian gland dysfunction: executive summary. Investigative ophthalmology \& visual science 52, 1922-1929, doi:10.1167/iovs.10-6997a (2011).

8. Zheng, Y., Wu, X., Lin, X. \& Lin, H. The Prevalence of Depression and Depressive Symptoms among Eye Disease Patients: A Systematic Review and Meta-analysis. Scientific reports, 7, 46453 https://doi.org/10.1038/srep46453 (2017).

9. Foulks, G. N. \& Borchman, D. Meibomian gland dysfunction: the past, present, and future. Eye \& contact lens, 36, 249-253 https://doi.org/10.1097/ICL.0b013e3181 ef0d37 (2010).

10. Amano, S. \& Inoue, K. Estimation of Prevalence of Meibomian Gland Dysfunction in Japan., 36, 684$688 \mathrm{https}: / /$ doi.org/10.1097/IC0.0000000000001208 (2017).

11. Siak, J. J. K. et al. Prevalence and risk factors of meibomian gland dysfunction: the Singapore Malay eye study., 31, 1223-1228 https://doi.org/10.1097/IC0.0b013e31823f0977 (2012).

12. Lekhanont, K., Rojanaporn, D., Chuck, R. S. \& Vongthongsri, A. Prevalence of dry eye in Bangkok, Thailand., 25, 1162-1167 (2006).

13. Yu, K. et al. Altered spontaneous activity in the frontal gyrus in dry eye: a resting-state functional MRI study. Scientific reports, 11, 12943 https://doi.org/10.1038/s41598-021-92199-8 (2021).

14. Andrianopoulou, A. et al. Functional connectivity and microstructural changes of the brain in primary Sjögren syndrome: the relationship with depression. Acta Radiol, 61, 1684-1694 https://doi.org/10.1177/0284185120909982 (2020). 
15. Ulusoy, M. O., Işık-Ulusoy, S. \& Kıvanç, S. A. Evaluation of dry eye disease in newly diagnosed anxiety and depression patients using anterior segment optical coherence tomography. Eye Vis (Lond), 6, 25 https://doi.org/10.1186/s40662-019-0149-y (2019).

16. Apkarian, A. V., Bushnell, M. C., Treede, R. D. \& Zubieta, J. K. Human brain mechanisms of pain perception and regulation in health and disease. Eur J Pain, 9, 463-484 (2005).

17. Kawashima, M. et al. Associations between subjective happiness and dry eye disease: a new perspective from the Osaka study. PloS one, 10, e0123299 https://doi.org/10.1371/journal.pone.0123299 (2015).

18. Koçer, E., Koçer, A., Özsütçü, M., Dursun, A. E. \& Krpnar, Ä. Dry Eye Related to Commonly Used New Antidepressants. J Clin Psychopharmacol, 35, 411-413 https://doi.org/10.1097/JCP.0000000000000356 (2015).

19. Zhang, X., Yin, Y., Yue, L. \& Gong, L. Selective Serotonin Reuptake Inhibitors Aggravate DepressionAssociated Dry Eye Via Activating the NF-KB Pathway. Investigative ophthalmology \& visual science, 60, 407-419 https://doi.org/10.1167/iovs.18-25572 (2019).

20. Wang, Z., Yang, H., Guo, Z., Liu, B. \& Geng, S. Socio-demographic characteristics and co-occurrence of depressive symptoms with chronic diseases among older adults in China: the China longitudinal ageing social survey. BMC Psychiatry, 19, 310 https://doi.org/10.1186/s12888-019-2305-2 (2019).

21. Kerner, N. A. \& Roose, S. P. Obstructive Sleep Apnea is Linked to Depression and Cognitive Impairment: Evidence and Potential Mechanisms. Am J Geriatr Psychiatry, 24, 496-508 https://doi.org/10.1016/j.jagp.2016.01.134 (2016).

22. Cheng, B. T. \& Silverberg, J. I. Depression and psychological distress in US adults with atopic dermatitis. Ann Allergy Asthma Immunol, 123, 179-185 https://doi.org/10.1016/j.anai.2019.06.002 (2019).

23. Read, J. R., Sharpe, L., Modini, M. \& Dear, B. F. Multimorbidity and depression: A systematic review and meta-analysis. J Affect Disord, 221, 36-46 https://doi.org/10.1016/j.jad.2017.06.009 (2017).

24. Tsang, A. et al. Common chronic pain conditions in developed and developing countries: gender and age differences and comorbidity with depression-anxiety disorders. J Pain, 9, 883-891 https://doi.org/10.1016/j.jpain.2008.05.005 (2008).

25. Yucekul, B., Mocan, M. C., Kocabeyoglu, S., Tan, C. \& Irkec, M. Evaluation of Long-Term Silicone Hydrogel Use on Ocular Surface Inflammation and Tear Function in Patients With and Without Meibomian Gland Dysfunction. Eye \& contact lens, 45, 61-66 https://doi.org/10.1097/ICL.0000000000000526 (2019).

26. Al-Dairi, W., Sowayigh, A., Alkulaib, O. M., Alsaad, A. \& N. S. \& The Relationship of Dry Eye Disease With Depression in Saudi Arabia: A Cross-Sectional Study. Cureus, 12, e12160 https://doi.org/10.7759/cureus.12160 (2020).

27. Gaudiani, J. L., Braverman, J. M., Mascolo, M. \& Mehler, P. S. Ophthalmic changes in severe anorexia nervosa: a case series. Int J Eat Disord, 45, 719-721 https://doi.org/10.1002/eat.20970 (2012). 
28. Sherwin, J. C., Reacher, M. H., Dean, W. H. \& Ngondi, J. Epidemiology of vitamin A deficiency and xerophthalmia in at-risk populations. Trans $R$ Soc Trop Med Hyg, 106, 205-214 https://doi.org/10.1016/j.trstmh.2012.01.004 (2012).

29. Ayaki, M. et al. Sleep Disorders are a Prevalent and Serious Comorbidity in Dry Eye. Investigative ophthalmology \& visual science, 59, DES143-DES150 https://doi.org/10.1167/iovs.17-23467 (2018).

30. Wu, M., Liu, X., Han, J., Shao, T. \& Wang, Y. Association Between Sleep Quality, Mood Status, and Ocular Surface Characteristics in Patients With Dry Eye Disease., 38, 311-317 https://doi.org/10.1097/IC0.0000000000001854 (2019).

31. van Leeuwen, N. et al. Crying Without Tears: Dimensions of Crying and Relations With Ocular Dryness and Mental Well-Being in Patients With Sjögren's Syndrome. J Clin Psychol Med Settings, 23, 77-87 https://doi.org/10.1007/s10880-015-9432-9 (2016).

32. Gomes, J. A. P. \& Santo, R. M. The impact of dry eye disease treatment on patient satisfaction and quality of life: A review. The ocular surface, 17, https://doi.org/10.1016/j.jtos.2018.11.003 (2019).

33. Tomlinson, A. et al. The international workshop on meibomian gland dysfunction: report of the diagnosis subcommittee. Investigative ophthalmology \& visual science 52, 2006-2049, doi:10.1167/iovs.10-6997f (2011).

34. Wang, Y. X., Wei, W. B., Xu, L. \& Jonas, J. B. Physical activity and eye diseases. The Beijing Eye Study. Acta ophthalmologica, 97, 325-331 https://doi.org/10.1111/aos.13962 (2019).

35. Labbé, A. et al. Dry eye disease, dry eye symptoms and depression: the Beijing Eye Study. The British journal of ophthalmology, 97, 1399-1403 https://doi.org/10.1136/bjophthalmol-2013-303838 (2013).

36. Zhang, H., Li, H., Ma, Q., Yang, F. Y. \& Diao, T. Y. Predicting malignant transformation of esophageal squamous cell lesions by combined biomarkers in an endoscopic screening program. World $\mathrm{J}$ Gastroenterol, 22, 8770-8778 (2016).

37. Garza-Leon, M., Gonzalez-Dibildox, A., Ramos-Betancourt, N. \& Hernandez-Quintela, E. Comparison of meibomian gland loss area measurements between two computer programs and intra-inter-observer agreement. Int. Ophthalmol, 40, 1261-1267 https://doi.org/10.1007/s10792-020-01292-w (2020).

38. Knop, E., Knop, N., Millar, T., Obata, H. \& Sullivan, D. A. The international workshop on meibomian gland dysfunction: report of the subcommittee on anatomy, physiology, and pathophysiology of the meibomian gland. Investigative ophthalmology \& visual science 52, 1938-1978, doi:10.1167/iovs.10-6997c (2011).

\section{Figures}


A Study flow of multi-center cross-sectional study

\section{Study Flow}

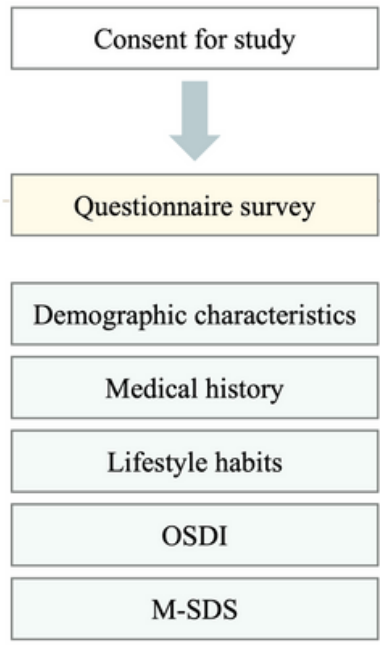

Data synthesis and analysis

\begin{tabular}{|c|c|}
\hline \multicolumn{2}{|c|}{ Ocular assessment } \\
\hline BCVA & IOP \\
\hline $\begin{array}{c}\text { Lid } \\
\text { abnormality }\end{array}$ & $\begin{array}{c}\text { MG } \\
\text { expressibility }\end{array}$ \\
\hline LLT & MG Loss \\
\hline TBUT & PB \\
\hline $\begin{array}{l}\text { Corneal } \\
\text { Staining }\end{array}$ & Schirmer I \\
\hline
\end{tabular}

B Distribution of participant units

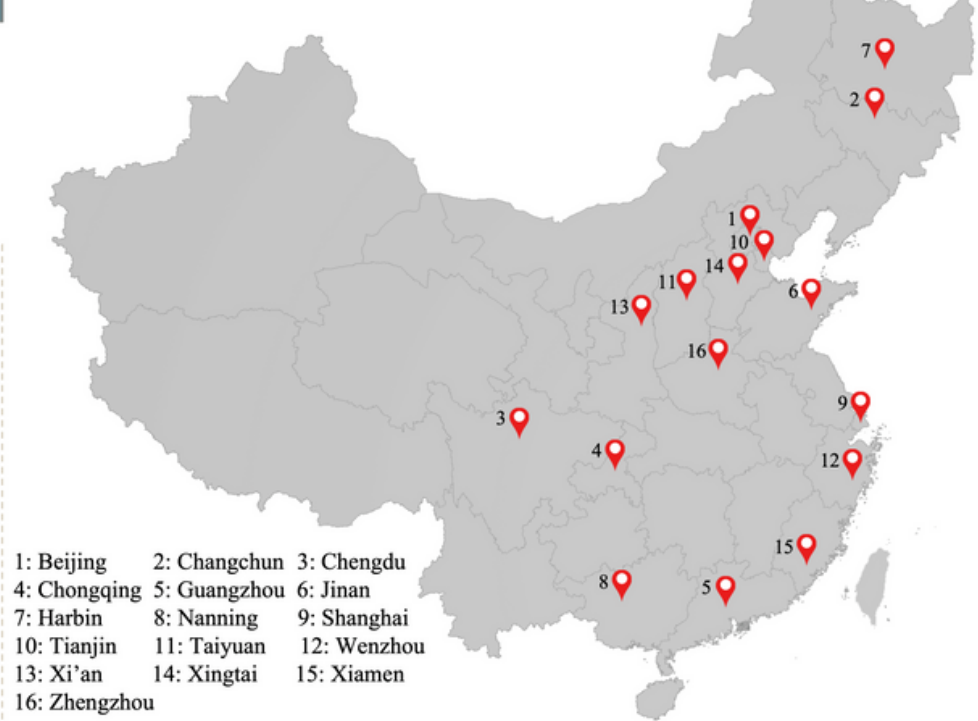

\section{Figure 1}

The study flow (Part A) and the geographic distribution of 23 eye centers (Part B). OSDI: ocular surface disease index; M-SDS: modified self-rating depression scale; BCVA: best corrected visual acuity; IOP: intraocular pressure; LLT: lipid layer thickness; MG: meibomian gland; TBUT: tear film break-up time; PB: partial blink. (The figure used with permission from co-author $\mathrm{Cao}$ )

\section{Supplementary Files}

This is a list of supplementary files associated with this preprint. Click to download.

- SupplementaryTable1.docx 